\title{
Histological Changes in Stems of Pinus sylvestris Seedlings Infected with a Virulent Isolate of the Pinewood Nematode Bursaphelenchus xylophilus
}

\author{
Inês Vieira da Silva ${ }^{1}$, Pedro Barbosa ${ }^{2}$, Manuel Mota $^{2}$ and Lia Ascensão ${ }^{1}$ \\ ${ }^{1 .}$ Centro de Biotecnologia Vegetal (IBB), Departamento de Biologia Vegetal, Faculdade de Ciências, \\ Universidade de Lisboa, Campo Grande, 1749-016 Lisboa, Portugal. \\ 2. NemaLab/ICAAM, Instituto de Ciências Agrárias e Ambientais Mediterrânicas, Universidade de Évora, \\ Núcleo da Mitra, 7002-554 Évora, Portugal.
}

Bursaphelenchus xylophilus (Steiner and Buhrer) Nickle, the pinewood nematode (PWN) responsible for pine wilt disease (PWD), has become a serious threat to global pine forests. It is native from North America, where is not associated with any pathology, but when introduced in the Far East induced a disease with severe environmental and economic impacts. In Europe, PWN was first detected in maritime pine, Pinus pinaster Aiton, at Setubal peninsula (Portugal) and, currently all the country is considered a quarantine area for PWN, representing an increasingly risk of spread to European forests. The vector, a cerambycid beetle, Monochamus galloprovincialis Olivier, carries the nematode from injured to healthy trees, through feeding wounds. After invading pine trees, nematodes feed from the plant tissues and multiply rapidly [1 and ref. cit. therein]. Although extensive research has been conducted in this disease, particularly in countries in which their own forest have been devastated, a detailed mechanism of the PWN invasion is still not well described. Therefore, the present work aimed to study the histological changes induced by B. xylophilus in Pinus sylvestris L. seedling tissues.

Two-year-old $P$. sylvestris seedlings were inoculated in scratched stems with 2,000 PWNs (mixed stages) from a virulent Portuguese isolate (HF). Small portions of stems from control and infected pine seedlings were collected at different time points until 7 weeks after inoculation. Samples were fixed in buffered glutaraldehyde/paraformaldehyde and prepared for scanning electron microscopy (SEM) or embedding in Leica Historesin ${ }^{\circledR}$, following standard methods. Historesin sections were stained with PAS/Toluidine Blue O and PAS/Comassie Blue.

The initial symptoms of disease, needle yellowing and/or browning, began about 1-2 weeks after inoculation. Although the development of symptoms varied among the specimens, seven weeks after inoculation the seedlings looked dried and dead. By contrast no external disease symptoms were observed in control seedlings. Stem cross sections of control seedlings showed the characteristic histological features of pine shoots. A variable number of axial resin ducts were distributed in the cortex, primary and secondary xylem (Fig. 1A, B), whereas radial resin ducts occurred in the xylem rays extending from secondary xylem to the cortex (data not shown). At the "early stage" of infection, when no external symptoms were visible the number of tanniniferous idioblasts increased as a response to wound and infection. Nematodes were firstly observed within the lumen of the cortex resin ducts. During the "advanced stage" of infection, when external symptoms were plainly visible, severe degradation in all stem tissues was evident (Fig. 1C, D). Cavities with irregular boundaries developed from degraded resin ducts and surrounding parenchyma cells. Nematodes were found in the xylem axial and radial ducts and their number per duct increased markedly. As the disease progressed the cambial zone underwent degradation and the cavities along this area enlarged and fused. At the fifth week after inoculation the number of nematodes increased dramatically and all stem tissues were severely damaged. Seven weeks after inoculation, the infected zone above the inoculation point and the close region above were dried and dead. Similar histological features were reported for the Asiatic susceptible pine 
species (e.g. $P$. thunbergii, $P$. densiflora) $[2,3,4]$. The wilting process of PWD has been explain, at least in part, by water deficit induced by nematodes pathological cavitation. However, a growing body of evidence indicates that the disease is the result of a complex interaction among nematodes, bacteria and fungi [5]. Further research will be needed to a better understand of all these relationships [6].

\section{References:}

[1] K Futai, Annual Review of Phytopathology 51 (2013), p. 61.

[2] N Hara et al, Japanese Journal of Nematology 36 (2006), p. 23.

[3] Y Mamiya in "Pine Wilt Disease: A Worldwide Threat to Forest Ecosystems", eds. M M Mota and P R Vieira (Springer, Berlin) p. 321.

[4] Y Mori et al, European Journal of Plant Pathology 122 (2008), p. 529.

[5] Z Wang et al, Journal of Microbiology 4 (2010), p. 437.

[6] Study financially supported by Fundação para a Ciência e Tecnologia (FCT) under the research contract PTDC/AGR-CFL/120184/2010 (ref. COMPETE FCOMP-01-0124-FEDER-019454).

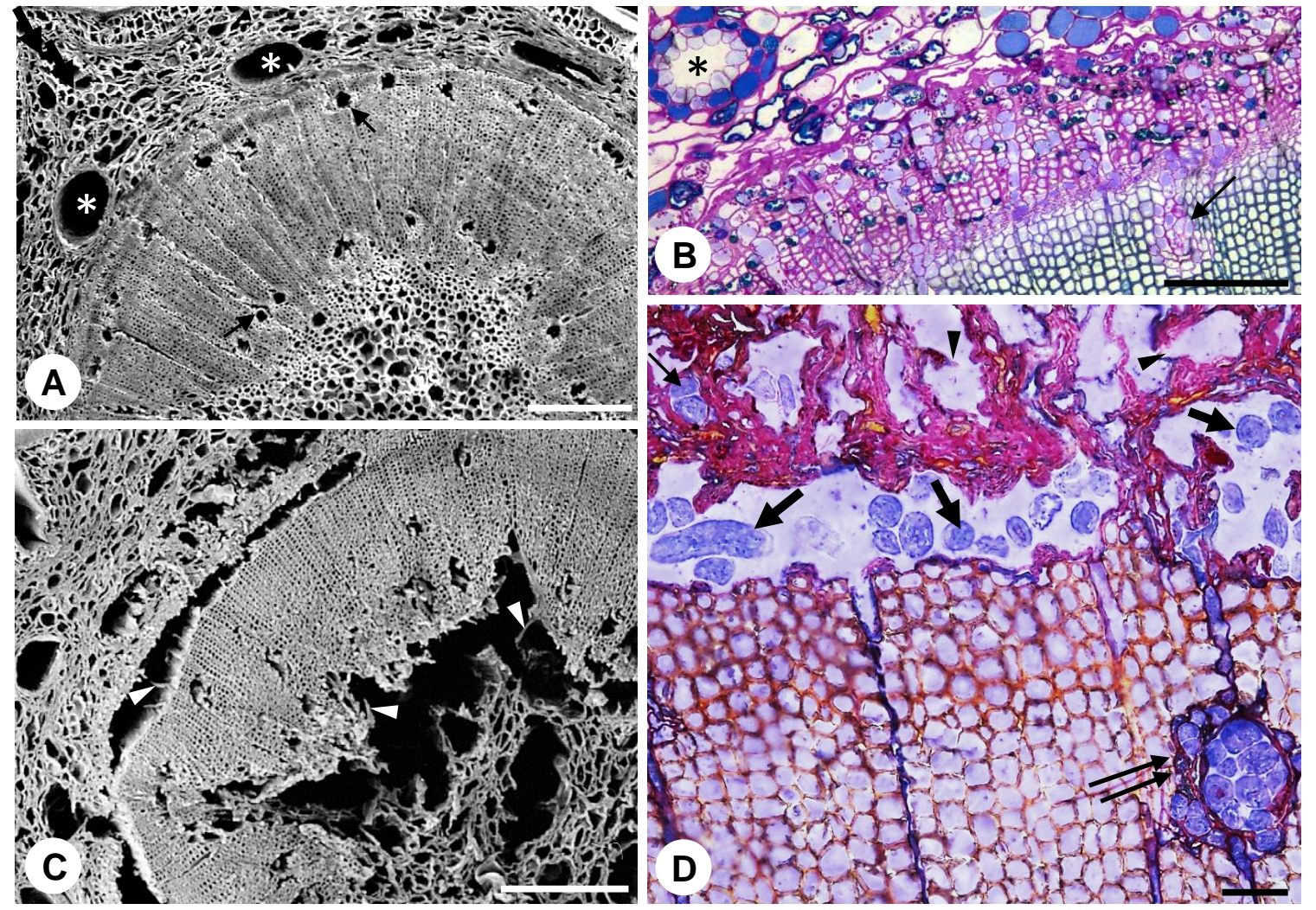

Figure 1. SEM micrographs (A, C) and light micrographs (B, D) of stem cross-sections from two year-old Pinus sylvestris seedlings. A, B, Control plants. Cortical resin ducts (asterisks) and axial resin ducts (arrows) surrounded by intact epithelial cells were observed. No signals of cell degradation were found. C, D, Bursaphelenchus xylophilus infected plants five weeks after inoculation. Resin ducts epithelial cells were destroyed. Broken and degraded cell walls (arrowheads) and cavities with irregular boundaries, were clearly seen. In D, nematodes can be observed within cavities in the cortex (arrow), in the cambial region (bold arrows) and in axial resin ducts (double arrow).

Bars $=80 \mu \mathrm{m}(\mathrm{A}, \mathrm{C})$ and $30 \mu \mathrm{m}(\mathrm{B}, \mathrm{D})$. 